\title{
Os perigos dos agrotóxicos para famílias agricultoras - Uma revisão integrativa
}

\author{
The dangers of pesticides for farming families - An integrative review \\ Los peligros de los plaguicidas para las familias agricultoras: Una revisión integradora
}

Recebido: 19/07/2021 | Revisado: 25/07/2021 | Aceito: 27/07/2021 | Publicado: 03/08/2021

Pedro Henrique da Silva Monteiro

ORCID: https://orcid.org/0000-0002-4951-4235

Universidade Tecnológica Federal do Paraná, Brasil

E-mail: pedromonteiro@alunos.utfpr.edu.br

Larisse Medeiros Gonçalves

ORCID: https://orcid.org/0000-0002-8546-3244

Universidade Tecnológica Federal do Paraná, Brasil

E-mail: larisse@alunos.utfpr.edu.br

Luana Santos dos Santos

ORCID: https://orcid.org/0000-0001-5771-9882 Universidade Tecnológica Federal do Paraná, Brasil E-mail: luana.1995@alunos.utfpr.edu.br

Lígia Kochhan de Fraga

ORCID: https://orcid.org/0000-0002-5311-6133 Universidade Tecnológica Federal do Paraná, Brasil E-mail: ligia.fraga@ifpr.edu.br

Marlete Turmina Outeiro

ORCID: https://orcid.org/0000-0002-3223-7649 Universidade Tecnológica Federal do Paraná, Brasil E-mail: marlete.outeiro@gmail.com

Adriane Rodrigues Zboralski

ORCID: https://orcid.org/0000-0001-8608-2163 Universidade Tecnológica Federal do Paraná, Brasil E-mail: adrianezboralski1@gmail.com

Wilson Itamar Godoy

ORCID: https://orcid.org/0000-0003-0152-6269

Universidade Tecnológica Federal do Paraná, Brasil E-mail: wigodoy@professores.utfpr.edu.br

\begin{abstract}
Resumo
A agricultura familiar é uma estrutura complexa e desempenha um papel fundamental nos cenários socioeconômico e ambiental do mundo inteiro, contudo, a maioria dos agricultores estão sujeitos ao desemparo público e a muitos fatores de riscos. Esse estudo tem como objetivo analisar e debater questões sobre os riscos do uso de agrotóxicos, especialmente na agricultura familiar. Para a realização da pesquisa foi aplicada a metodologia de revisão integrativa, que fornece amplas informações sobre um assunto/problema, entrelaçando a base conceitual com o que há de mais recente. Os resultados demonstraram que agricultores familiares estão mais vulneráveis aos prejuízos causados por agrotóxicos que outros grupos rurais. Parte desses atores sociais desconhecem os danos que esses produtos podem causar ou não possuem equipamentos adequados para se protegerem. As maiores exposições a esses riscos são encontradas em países em desenvolvimento. As notificações anuais de intoxicação não podem ser consideradas números absolutos, pois muitos casos acontecem em regiões com difícil acesso as informações. Considera-se que para uma mudança de panorama, deve-se: apoiar-se em outros estilos de agricultura mais alternativas, tornando o funcionamento dos agroecossistemas mais resilientes e ecológicos, que as entidades responsáveis tenham providências fundamentadas em abordagens mais integradas, que considerem o contexto socioeconômico, ambiental e de saúde em que estes então inseridos, bem como promovam informações para o campo, visando a qualidade de vida dos indivíduos, tão importantes para sociedade.
\end{abstract}

Palavras-chave: Agroquímicos; Contaminação; Meio ambiente e saúde.

\begin{abstract}
Family farming is a complex structure and plays a key role in socioeconomic and environmental settings worldwide, yet most farmers are subject to public neglect and many risk factors. This study aims to analyze and debate issues about the risks of pesticide use, especially in family farming. To carry out the research, the integrative review methodology was applied, which provides ample information about a subject/problem, interweaving the conceptual basis with the latest developments. The results showed that family farmers are more vulnerable to the damage caused by pesticides than other rural groups. Some of these social actors are unaware of the damage that these products can cause or do not have adequate equipment to protect themselves. The highest exposures to these risks are found in
\end{abstract}


developing countries. The annual poisoning notifications cannot be considered absolute numbers, because many cases happen in regions with difficult access to information. It is considered that for a change in the panorama, it is necessary to support other styles of more alternative agriculture, making the functioning of agro-ecosystems more resilient and ecological, that the responsible entities have measures based on more integrated approaches, which consider the socioeconomic, environmental and health context in which they are inserted, as well as promote information for the field, aiming at the quality of life of individuals, so important to society.

Keywords: Agrochemicals; Contamination; Environment and health.

\section{Resumen}

La agricultura familiar es una estructura compleja y desempeña un papel clave en los escenarios socioeconómicos y medioambientales de todo el mundo; sin embargo, la mayoría de los agricultores están sujetos a la indefensión pública y a muchos factores de riesgo. Este estudio pretende analizar y debatir cuestiones sobre los riesgos del uso de plaguicidas, especialmente en la agricultura familiar. Para llevar a cabo la investigación, se aplicó la metodología de la revisión integradora, que proporciona amplia información sobre un tema/problema, entrelazando la base conceptual con lo más reciente. Los resultados mostraron que los agricultores familiares son más vulnerables a los daños causados por los pesticidas que otros grupos rurales. Algunos de estos actores sociales no son conscientes de los daños que pueden causar estos productos o carecen del equipo adecuado para protegerse. La mayor exposición a estos riesgos se encuentra en los países en desarrollo. Los informes anuales sobre intoxicaciones no pueden considerarse cifras absolutas, ya que muchos casos se producen en regiones con difícil acceso a la información. Se considera que para un cambio en el panorama, se debe apoyar en otros estilos de agricultura más alternativos, haciendo que el funcionamiento de los agroecosistemas sea más resiliente y ecológico, que las entidades responsables tengan medidas basadas en enfoques más integrados, que consideren el contexto socioeconómico, ambiental y de salud en el que se insertan, así como promover la información al campo, apuntando a la calidad de vida de los individuos, tan importante para la sociedad.

Palabras clave: Agroquímicos; Contaminación; Medio ambiente y salud.

\section{Introdução}

O universo agrário é complexo, a sua diversidade abraça vários grupos, objetivos, interesses, etc. A agricultura familiar (AF), por exemplo, pode ser considerada um elemento chave da diversidade desse ramo de trabalho (Wanderley, 2009). A AF desempenha diversas funções sociais, ambientais e econômicas, em nível local, regional e nacional, sendo base para produção e comércio alimentar.

A Food and Agriculture Organization (FAO) (2014) afirma que a mesma detém cerca de 75\% dos recursos agrícolas no mundo, produzindo aproximadamente $80 \%$ dos alimentos consumidos. No Brasil, ela é responsável pela produção de $70 \%$ dos alimentos básicos consumidos pela população, atuando na cadeia produtiva de culturas como: mandioca (87\%), feijão (70\%), carne suína (59\%), leite (58\%), carne de aves $(50 \%)$ e milho $(46 \%)$, colaborando dessa forma para a segurança alimentar e nutricional do país (MDA, 2016).

Uma das estratégias empregadas na produção de alimentos da AF é a aplicação de insumos agrícolas, como por exemplo os agrotóxicos. Eles podem ser conceituados como produtos químicos-sintéticos manuseados nos diversos meios das agriculturas e pecuária, sejam elas de grande escala ou menores (Feitosa et al., 2020). Os agrotóxicos podem ser classificados em: inseticidas, fungicidas, acaricidas, nematicidas, herbicidas, bactericidas, vermífugos, estes podem ser solventes, tintas, lubrificantes, produtos para limpeza e desinfecção de estábulos (Braibante \& Zappe, 2012).

Estes agroquímicos possuem a função de controlar, prevenir, destruir ou repelir qualquer forma de agente patogênico, animal ou vegetal que seja danoso às produções (Bohner et al., 2013). Apesar desse item ter função de proteção contra pragas, doenças e plantas daninhas, se forem aplicados de maneira desordenada e sem o uso de equipamentos adequados podem resultar em grandes problemáticas para o meio ambiente e para saúde dos seres humanos (Lopes, 2017).

De acordo com análises realizadas pelo Programa de Análise de Agrotóxicos em Alimentos, mais de 30\% dos alimentos diariamente consumidos no Brasil encontram-se contaminados por agrotóxicos (Anvisa, 2011). De acordo com o dossiê publicado pela Associação Brasileira de Saúde Coletiva (Abrasco) (Carneiro, 2015), a contaminação dos alimentos vincula-se à utilização de quantidades significativamente superior ao recomendado, tanto no que se refere ao quantitativo de 
doses por aplicação quanto ao número de aplicações.

Nesse contexto, a exposição humana a agrotóxicos deve ser uma preocupação de saúde pública, em especial, nos países em desenvolvimento, pois estes são os que mais sofrem com as consequências, visto que, existe uma falta de informação na sua utilização (Fao, 2020). Há necessidade de uma análise mais crítica e aberta do que a apresentada pelos fabricantes, pois os mesmos não avaliam a vulnerabilidade dos usuários e os diversos ambientes técnicos de sua inserção.

Esse tema possui grande relevância, por razões de saúde pública, meio ambiente e sustentabilidade da agricultura, em função do elevado crescimento da produção agrícola e do uso de agrotóxicos no país (IPEA, 2019). O objetivo desse artigo é analisar e debater questões importantes acerca dos riscos da utilização de agrotóxicos nas produções de alimentos, especialmente, pelos agricultores familiares.

\section{Metodologia}

Para a realização deste estudo foi aplicada a metodologia de revisão integrativa, que consiste em estratégias científicas de agregar teorias, resultados e experiências de maneira sistemática, entretanto de forma ordenada e abrangente, ela fornece informações mais amplas sobre um assunto/problema, compondo um corpo de um eixo do tema com literatura base e estudos atuais. Dessa forma, ela se torna uma integração do compilado de informações, abordando da teoria à prática (Ercole et al., 2014).

As buscas foram realizadas principalmente nas bases de dados eletrônicas Google Scholar ${ }^{\circledR}$, Sciencedirect ${ }^{\circledR}$, Springer ${ }^{\circledR}$, Scopus ${ }^{\circledR}$. A amplitude de data dos trabalhos abordados para elaboração dessa pesquisa variou de 1989 a 2020. Para escolha dos artigos, adotou-se os critérios: datas atuais, autores que são referência da área, além de analisar detalhadamente se o contexto dos estudos era relevante para o tema proposto, deu-se preferência à publicação em periódicos, notas técnicas de intuições do ramo e matérias publicadas em jornais respaldados.

Salienta-se que artigos com publicação acima de 10 anos são de cunho conceitual fundamentais para dar apoio a pesquisa, principalmente, no que tange o aporte conceitual. O tema escolhido foi o risco dos agrotóxicos para agricultura familiar e foram subdivididos nos seguintes tópicos: conceitos de embasamento para a discussão acerca da problemática em foco e risco dos agrotóxicos para as famílias rurais.

Foram selecionados 50 documentos, entre eles, artigos, livros, leis e informativos técnicos. A estruturação dos resultados se deu pela seguinte ondem: a) base conceitual, abrangendo o histórico, legislação, classificação toxicológica, definições e o fechamento da sessão com dados de consumo no Brasil; b) Importância da agricultura familiar, dados institucionais que registram casos de intoxicações, pesquisas científicas, nacionais e internacionais que discorram acerca da problemática em determinadas delimitações geográficas.

\section{Resultados e Discussão}

\subsection{Conceitos Base Para a Discussão Acerca da Problemática em Foco}

O ser humano sempre buscou formas para conter as dificuldades naturais existentes nas lavouras, afim de aumentar e melhorar a produtividade. Em alguns casos, a história registra a realização de cerimônias religiosas e rituais de magia como método de controle ou extinção de pragas (Pavani, 2016).

Os gregos e romanos, por exemplo, acreditavam em deuses específicos que protegiam os seus cultivos de pragas e doenças. Durante o período clássico, mesmo com o escasso conhecimento sobre as leis da natureza e das pragas que devastavam as áreas agrícolas, já existiam indícios de uso de métodos para o combate de pragas. Na idade média houve pouca evolução a respeito de conhecimento científico, acreditava-se que a justiça deveria ser realizada para garantir a prevalência do bem e a punição dos infratores. Este pensamento contribuiu para o surgimento das práticas de julgamento de pragas em 
tribunais eclesiásticos (Braibante \& Zappe, 2012; Pavani, 2016).

Com o desenvolvimento do conhecimento científico e através dos métodos de observação, tentativa e erro, descobriuse inúmeros compostos químicos eficientes no controle de insetos e fungos. Contudo, ainda eram vagas as informações sobre as composições e fórmulas químicas das substâncias utilizadas. Em razão das mudanças nos processos produtivos e a utilização em larga escala de agroquímicos, durante a metade do século XIX as dificuldades com as pragas se agravaram, impulsionando as primeiras pesquisas científicas criteriosas a respeito da utilização de compostos químicos, com a finalidade de conter pragas agrícolas. Nesta época, também já eram empregados compostos inorgânicos e extratos vegetais (Barbosa, 2004; Braibante \& Zappe, 2012).

Na metade século XX, sugiram muitas inovações tecnológicas impulsionadas pelo fenômeno da revolução verde, responsável por gerar grandes modificações na paisagem natural, especialmente no meio agrícola. Essas tecnologias inovadoras resultaram na troca da mão de obra humana e tração animal pelos implementos agrícolas, sementes com elevado potencial produtivo e recentemente, no desenvolvimento das sementes transgênicas. Neste âmbito também ocorreu a intensificação da aplicação de agrotóxicos e de adubos químicos sintéticos (Borsoi et al., 2014).

Todos os conhecimentos e inovações serviram de base para o desenvolvimento das atuais técnicas agrícolas, na qual aumentar a eficiência produtiva para suprir as demandas globais por alimentos é o objetivo principal. No entanto, esses métodos de produção ocasionaram em uma dependência excessiva dos agrotóxicos, pouca preocupação com os procedimentos necessários para aplicação de forma segura e em inúmeras sequelas que essas substâncias podem causar à saúde dos seres vivos, em especial trabalhadores e a população em geral que é contaminada por quantidades significativas de resíduos de agrotóxicos existentes nos alimentos, na água, no ar e no solo (Borsoi et al., 2014).

Frisa-se que os inseticidas começaram a ser utilizados em grande escala na década de 1940, durante a Segunda Guerra Mundial, a fim de proteger os soldados das regiões tropicais e subtropicais da África e da Ásia, das pragas transmissoras da doença-do-sono, malária, dentre outras. Devido à necessidade de proteger o exército, as pesquisas de novos inseticidas foram impulsionadas, o que resultou no desenvolvimento de vários agrotóxicos que são usados ainda hoje (Braibante \& Zappe, 2012).

No Brasil, durante o período da revolução verde, foram implementadas muitas medidas públicas incentivando o emprego dos agrotóxicos, por consequência inúmeros agricultores em todo território nacional passaram a utilizar essa prática em suas áreas de cultivo. Deste modo, o uso de agroquímicos recebeu grande relevância em âmbito nacional, e em pouco tempo foi assimilada também pela $\mathrm{AF}$ com a finalidade de reduzir o surgimento intenso de pragas, que devastaram as lavoras por todo o país e diminuir as perdas econômicas, buscando assimilar o que era entendido como desenvolvimento (Costa \& Pires, 2016).

É importante ressaltar a conceituação do termo "agrotóxico" estabelecido pela Lei Federal no 7.802 de 11 de julho de 1989, artigo 2, inciso I, regulamentada pelo Decreto $n^{\circ} .98$ :

Os produtos e os componentes de processos físicos, químicos ou biológicos destinados ao uso nos setores de produção, armazenamento e beneficiamento de produtos agrícolas, nas pastagens, na proteção de florestas nativas ou implantadas e de outros ecossistemas e também em ambientes urbanos, hídricos e industriais, cuja finalidade seja alterar a composição da flora e da fauna, a fim de preservá-la da ação danosa de seres vivos considerados nocivos, bem como substâncias e produtos empregados como desfolhantes, dessecantes, estimuladores e inibidores do crescimento (Brasil, 1989).

Mesmo que a conceituação remeta a pontos benéficos, no decorrer dos tempos, percebeu-se que os agrotóxicos também causavam diversos efeitos danosos à saúde humana, os mesmos estão relacionados as suas características químicas, da quantidade absorvida ou ingerida, do tempo de exposição e das condições gerais de saúde de quem se contaminou (Dallefi \& 
Siqueira, 2017).

A classificação desses elementos pode resultar em diferentes classes toxicológicas, por meio de faixas coloridas (Figura 1). Essa classificação corresponde aos testes e estudos efetivados em laboratório que tentam instituir a dosagem letal (DL) do agrotóxico em 50\% dos animais utilizados naquela concentração (Inca, 2020).

Figura 1: Classificação dos agrotóxicos de acordo com os efeitos à saúde humana.

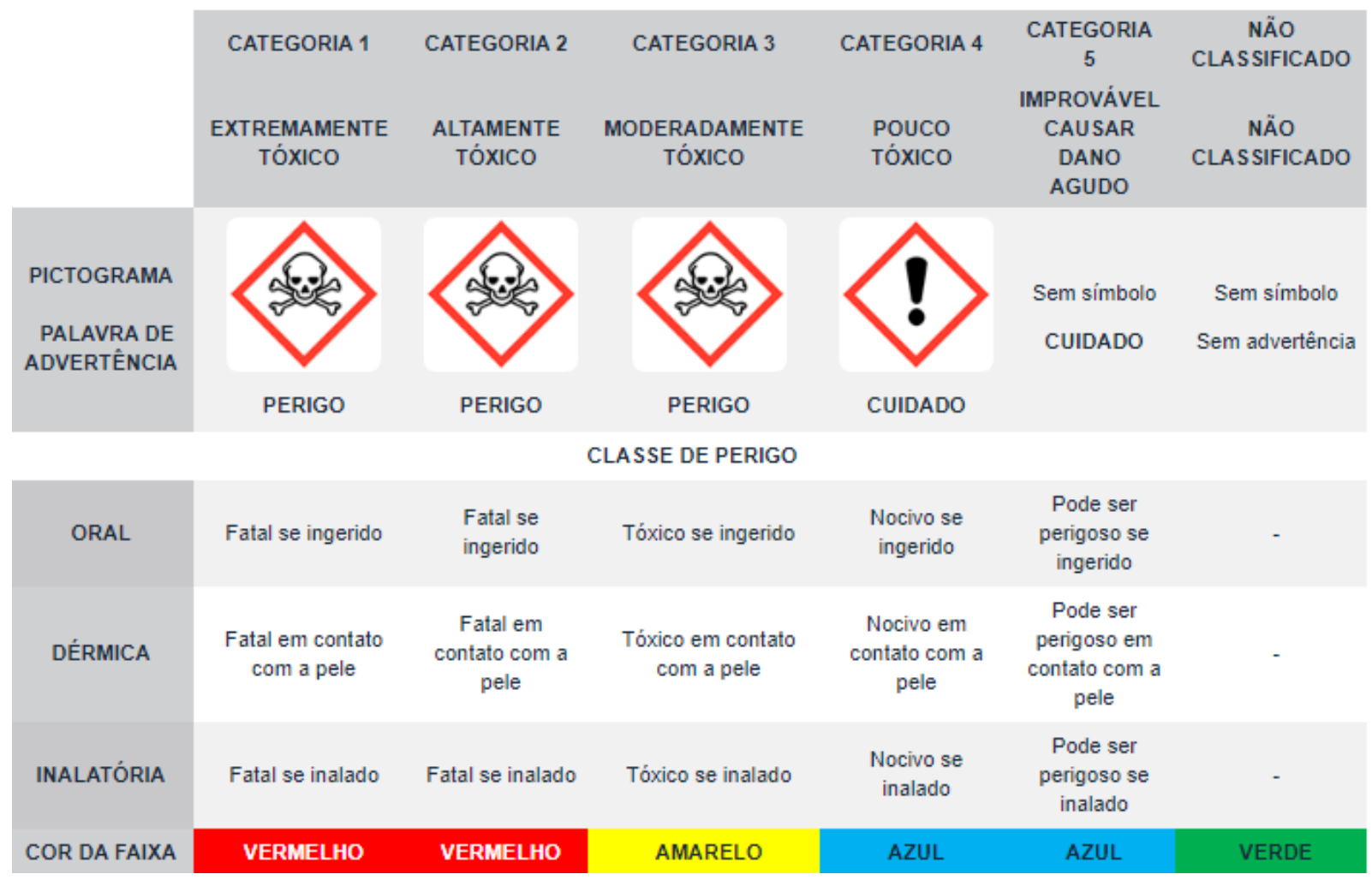

Fonte: Instituto Nacional do Câncer/ANVISA (2020) Agrotóxicos altamente perigosos.

São definidos FAO e World Health Organization (Who) (2014) como pesticidas reconhecidos por apresentarem níveis elevados de riscos à saúde (agudos ou crônico) e ao meio ambiente de acordo com sistemas de classificação do Código Internacional de Conduta sobre Gerenciamento de Pesticidas. Com o objetivo de reduzir os riscos de eventuais acidentes ou contaminação com essas substâncias, qualquer tipo de agrotóxico, independente da sua classe, deve ser manejado com precaução, obedecendo todas as indicações dos especialistas e fabricantes.

Neste sentido, é fundamental que os aplicadores utilizem os Equipamentos de Proteção Individual (EPI). Os EPIs são constituídos basicamente por touca árabe, respirador, viseira, jaleco, avental, calça, botas e luvas (Figura 2). Os EPIs além de serem indispensáveis para os profissionais que atuam diretamente com a pulverização nas lavouras, também é recomendado para a proteção de quem trabalha nos setores de transporte e armazenamento dos agroquímicos (Schlosser, 2017). 
Figura 2: Principais EPIs utilizados na aplicação de agroquímicos.

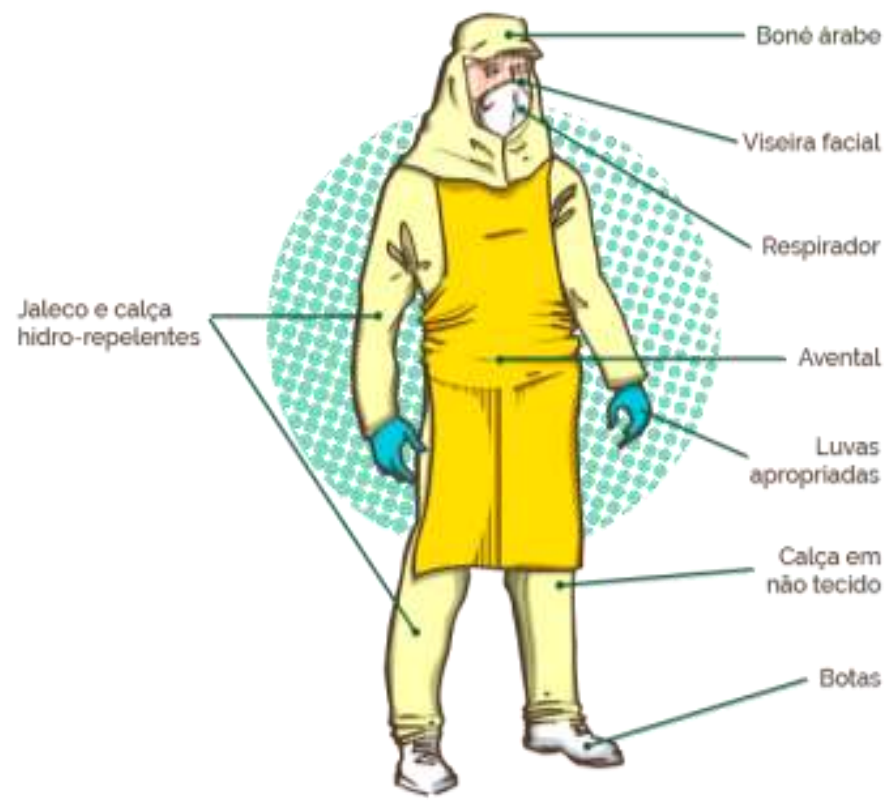

Fonte: Boas Práticas Agronômicas (2018).

Neste contexto, a Norma Regulamentadora (NR) 31, Portaria MTE n86, 2005, apresenta grande importância, visto que regulamenta a segurança no trabalho agrícola, e tem como objetivo principal de "estabelecer os preceitos a serem observadas na organização e no ambiente de trabalho, de forma a tornar compatível o planejamento e o desenvolvimento das atividades da agricultura, pecuária, silvicultura, exploração florestal e aquicultura com a segurança e saúde e meio ambiente do trabalho" (NR 31, 2005).

O item 31.8.1 da NR 31 considera em risco os trabalhadores em exposição direta, os que manuseiam os agrotóxicos em qualquer uma das etapas de armazenamento, transporte, preparo, aplicação, descarte, e descontaminação de equipamentos e vestimentas e também os trabalhadores em exposição indireta os que cercam e cumprem atividades de trabalho em áreas próximas de onde se faz a manipulação dos agrotóxicos em alguma das etapas citadas acima, e ainda os que desempenham atividades de trabalho em áreas recém-tratadas. Ainda, segundo a NR 31 (2005, item 31.8.1) “a empresa ou empregador rural deve dispor instruções satisfatórias aos que manipulam agroquímicos, adjuvantes e afins, e aos que desenvolvam qualquer atividade em áreas onde possa haver exposição direta ou indireta a esses elementos, assegurar as condições de segurança previstos".

Embora exista a NR 31 estruturada para o trabalho rural, no que tange a segurança destes trabalhadores, falta informação e assistência para os agricultores no uso desses insumos sintéticos. Santana et al. (2013) afirmam que uma das principais limitações é referente à leitura dos rótulos, o uso exacerbado de termos técnicos e a ausência de clareza nas informações e o uso de letras extremamente diminutas, o que prejudica o entendimento de quem usa, principalmente, atores sociais da AF.

Em geral, o agricultor que vai até as casas agropecuárias e solicita algum tipo de defensivo agrícola sem receituário agronômico, auxilia nesses perigos intensos causados pelos agrotóxicos por conta da falta de informação dos mesmos associado à frouxidão da legislação (Falcão et al., 2013). Isso retrata que a legislação está aquém e em detrimento da saúde desses trabalhadores.

Neste sentindo, expõe-se que o receituário agronômico é essencial, serve como uma receita de remédio, que explica 
quanto necessita no uso e por quanto tempo, entretanto, não há fiscalização da venda. A aplicação de leis e regulamentos relativos ao registro e comercialização parece ter certo grau de flexibilidade, pois tais produtos químicos podem ser obtidos sem prescrições agronômicas (Ramos et al., 2020).

Vale destacar que no ano de 2019, o país aprovou o registro de 474 agrotóxicos, o maior número registrado pelo Ministério da Agricultura nos últimos cinco anos, cuja comercialização e utilização aumentaram 200\% entre 2000 e 2012 (Mapa, 2019). Cerca de um terço da receita dos fabricantes de agroquímicos líderes mundiais vem de produtos classificados como "altamente perigosos", que são vendidos principalmente para países emergentes e pobres, como Brasil e Índia (Bbc, 2020).

Portanto, esse contato é um sério problema de saúde pública, principalmente nos países em desenvolvimento, que atingem muitos profissionais que lidam com esses produtos, membros de comunidades rurais e os consumidores dos alimentos (Sindag, 2017). Diante toda a contextualização, questiona-se: que perigos os agroquímicos apresentam para agricultura familiar?

\subsection{Risco dos Agrotóxicos Para as Famílias Agricultoras}

Antes de fomentar o debate sobre os riscos desses elementos em sistemas produtivos, é interessante destacar a grande importância que famílias agricultoras possuem para o sistema global. A AF possui uma configuração peculiar em sua forma de trabalhar e produzir, esta dinâmica ocorre em perímetros definidos, onde os processos realizados promovem uma maior interação familiar, que em grande parte, apresentam algum grau de parentesco e se relacionam com outras unidades familiares e grupos sociais, também se destacam pela ligação com a terra e os demais meios de produção (Schneider, 2016).

Dados da FAO (2020), afirmam que cerca de $90 \%$ dos 570 milhões de estabelecimentos rurais do mundo pertencem e são operadas pelas famílias. E a maioria delas é considerada de pequena escala, entretanto, possuem um papel fundamental para a soberania alimentar mundial. Embora a agricultura seja o principal enfoque dessas famílias, na maioria das vezes elas realizam outras atividades econômicas (geralmente informais) para contribuir com suas receitas. Ademais, estes atores sociais são de uma importância ímpar para fortalecer uma agricultura sustentável e enfrentar o desafio de produzir mais alimentos (FAO, 2020; Borges et al., 2020).

Entretanto, por conta da introdução e exigências de práticas do modelo produtivista, a AF passou a utilizar agroquímicos, buscando uma equidade produtiva comparada aos produtores de larga escala (Toledo \& Zonin, 2020). Diante desse fato, estas famílias ficaram muito expostas à contaminação por estes elementos, isso pode acontecer tanto por meio direto (1) ou exposição ambiental (2). O Inca (2020), descreve essas duas possibilidades como:

(1) Através da inalação, contato dérmico ou oral durante a manipulação, aplicação e preparo do aditivo químico, transporte e comércio de agrotóxicos. (2) Através das pulverizações aéreas que ocasionam a dispersão dessas substâncias pelo meio ambiente contaminando as áreas e atingindo a população. Consumo de alimentos e água contaminados. Outra forma é o contato com roupas dos trabalhadores com o agrotóxico (Inca, 2020, p.2)

Casos de envenenamento por pesticidas atingem aproximadamente 3 milhões de pessoas a cada ano, ocasionando cerca de 220.000 mortes. Esses dados possuem maior evidência em países em desenvolvimento e na zona rural (Who, 2020). A Who (2020) estimam que os agrotóxicos ocasionam cerca de 70 mil intoxicações agudas e crônicas anualmente que evoluem para óbito. Os sobreviventes, podem vir a sofrer de doenças agudas e crônicas. Também existe a estimativa, de que em países em desenvolvimento, a cada 4 horas um trabalhador agrícola vem a óbito devido a intoxicação por agrotóxicos (Porto, 2018).

Apesar das estimativas, não há números absolutos de quantas pessoas por ano sofrem com os efeitos na saúde relacionados aos agrotóxicos, isso se deve a vários motivos, incluindo a falta de definição de caso padronizada, especialmente 
em zonas que vivem famílias agricultoras de pequena escala (Who, 2020).

A exposição a longo prazo, mesmo que em pequenas quantidades, pode ter sérios efeitos adversos à saúde. Sintomas neurológicos como perda de memória, disfunção cognitiva, capacidade visual reduzida, alteração de humor e comportamento, resposta reduzida a estímulos e habilidades motoras reduzidas. Outras manifestações prováveis incluem alergias, asma, desregulação endócrina, câncer, problemas reprodutivos e transtorno do desenvolvimento fetal em grávidas (Upadhayay et al., 2020; Inca, 2020).

No Brasil, existem dois sistemas chaves para análise de dados de intoxicação e óbitos, que são o Sistema Nacional de Informações Tóxico-Farmacológicas (SINITOX) e o Sistema de Informação de Agravos de Notificação (SINAN). Na Tabela 1, disposta abaixo, tem-se os últimos dados, SINITOX para o ano de 2017 e o agrupamento de casos de 2007-2019 pelo SINAN.

Tabela 1. Sistemas e suas apresentações mais recentes por intoxicação.

\begin{tabular}{cccc}
\hline Instituições & Data dos dados & Localização & No de intoxicações $^{\text {(n) }}$ \\
\hline SINITOX & 2017 & Brasil & 3.379 \\
SINAN & $2007-2019$ & Brasil & 52.732 \\
\hline
\end{tabular}

Fonte: FIOCRUZ (2020) e DATASUS (2020).

No SINITOX foram registrados cerca de 3.379 casos de intoxicação. Esses casos, estão incluídos usos agrícolas em estabelecimentos rurais $(75.41 \%)$ e usos domésticos $(24.41 \%)$. É de extrema relevância ressaltar que os agrotóxicos compõem a segunda posição em número de registro de intoxicação no país, perdendo apenas para medicamentos, entretanto, quando é relacionado ao número de óbitos, ele praticamente dobra, comparado aos medicamentos (Fiocruz/Sinotox, 2020).

Um estudo que analisou dados de intoxicação por agrotóxicos atendidos em 2017 pelos Centros Brasileiros de Controle de Intoxicações (Cbci), constatou números maiores que os de SINOTOX (2020), foram identificados 3.826 pacientes intoxicados por agrotóxicos, dos quais 146 morreram (Okuyama et al., 2020). A comparação dos estudos corrobora com as afirmações da Who (2020), de que não há números absolutos para intoxicação por pesticidas, pois, sempre existirão, especialmente, famílias agricultoras sem acesso direto e rápido dos sistemas de saúde.

No Sinan (2020), disposto no sistema Datasus do Sistema Único de Saúde (SUS) do Brasil, foram constatados 52.732 entre os anos de 2007 e 2019, foram casos de acidentes de trabalho por intoxicações exógenas causados por agrotóxicos no Brasil, com presença significante de agricultores familiares. Destaca-se que o estado do Paraná, obteve a maior participação nesses números, com cerca de 7.589 casos e 2.220 mortes em decorrência de intoxicação por agrotóxico.

Esse fato pode ser justificado por conta de que, embora o estado do Paraná, possua grande representatividade da agricultura familiar, elas estão inseridas numa cultura de produção simplificada de grãos, que são extremamente dependentes de insumos agrícolas. O Ministério da Agricultura, Pecuária e Abastecimento (Mapa) e Companhia Nacional de Abastecimento (Conab) (2018) ressaltam que o Paraná está vinculado aos quatro estados (Mato Grosso, Paraná, Rio Grande do Sul e Goiás) que mais produzem grãos no país.

Isso demonstra o quão importante é que haja impulsos ao desenvolvimento sustentável em sistemas agroalimentares. Agroecossistemas manejados com base em processos ecológicos, são menos dependentes de insumos externos, possuem biodiversidade funcional, ajudam as famílias agricultoras a terem autonomia socioeconômica e qualidade ambiental. Os benefícios vão além, pois, promovem também a soberania alimentar (Tittonell, 2020; Gonçalves, 2020). Para maior força na urgência em transformar esses sistemas, deve-se pautar e fortalecer pesquisas que ajudem a esboçar o quão prejudicial para sociedade, como todo, são os usos indiscriminados de agroquímicos e investigações que validem a importância de 
agroecossistemas com manejos sustentáveis.

Acerca de alguns estudos, uma pesquisa evidenciou o consumo exagerado de agrotóxicos no estado do Rio de Janeiro. Ele representou um quadro de intoxicações "invisíveis" por conta de muitos agricultores serem familiares e se encontrarem isolados, e não apenas geograficamente, mas também com assistência técnica que auxilie os mesmos a manusearem estes insumos (Tourinho \& Portel, 2016).

O relatório Vigilância em Saúde de Populações Expostas a Agrotóxicos no Estado do Rio de Janeiro", divulgado pelo Ministério da Saúde em 2018, apontou que o território Fluminense ocupa a décima posição entre os estados que mais usam agrotóxicos no País. No entanto, o número de casos de intoxicação pelo RJ é difícil de estimar porque os relatórios do estado são claramente insuficientes (Brasil, 2018).

No estado de Kano, Nigéria, houve uma análise das práticas de segurança e os comportamentos dos agricultores adotados no manuseio de pesticidas. Os resultados foram alarmantes, os principais dados apontam que $29,1 \%$ dos entrevistados armazenam agrotóxicos em seus quartos, cerca de 47,0\% descartam embalagens de pesticidas jogando em campo aberto, $72,6 \%$ misturam os agrotóxicos, entre outras situações. As considerações refletidas nesse estudo, foram de que as regulamentações de pesticidas para os agricultores devem ser aplicadas de forma a proteger a saúde pública e aqueles que estão sendo expostos as falhas dessas instruções, ainda, deve-se promover medidas de formação participativa sobre o potencial de propriedades perigosas (Olalekan et al., 2020).

Alguns estudos brasileiros, também são compatíveis com esses resultados, como em Santa Maria de Jetibá (ES). Como por exemplo, cerca de $89 \%$ dos agricultores entrevistados faziam uso de agrotóxicos extremamente tóxicos e $68 \%$ faziam misturas aleatórias, 59\% não liam os rótulos dos produtos e 71,4\% não usavam os EPIs ou utilizavam incorretamente (Petarli et al., 2019). Outra pesquisa analisou capacitação técnica e percepção de risco na saúde ocupacional de trabalhadores rurais expostos aos agrotóxicos no município de Cerro Largo, RS. Os autores verificaram que uma grande parcela dos agricultores recebeu treinamento de empresas que comercializam agrotóxicos. Os agricultores conhecem os perigos dessas substâncias, contudo, relatam ter indícios de intoxicação (Ristow et al., 2020).

Essas pesquisas corroboram com as informações da WHO (2020), sobre a desinformação e, consequentemente, os riscos, presentes em países em desenvolvimento. Inclusive, o corpo de artigos científicos acerca de problemas de saúde de agricultores familiares e a relação com uso de agrotóxico são expressivos nestes países. Pode-se citar os Abbasi-Jorjandi et al. (2020), Silvério et al. (2020), Mehmood (2020), Bach et al. (2020), Andin et al. (2020), entre outros.

O fato é que, infelizmente, além da exposição a estes elementos tóxicos no trabalho de famílias agricultoras este não é o único perigo, pois elas também podem servir de meio para cometer suicídios, diante à problemas de saúde mental que tem aumentado em todo mundo, tanto nas regiões urbanas quanto rurais (Wang, 2020).

Uma pesquisa, buscou fazer a relação do perigo da exposição de agrotóxicos e a prevalência de transtornos mentais comuns entre agricultores familiares no Rio de Janeiro, os resultados obtidos demonstraram que existem tendências à cenários de suicídios, sendo muito perigoso essas pessoas terem acesso a estas substâncias de forma facilitada. O estudo enfatiza que nos últimos anos, tem sido crescente o diagnóstico de doenças neurológicas (depressão, ansiedade, síndrome do pânico, entre outros) no meio rural, especialmente, na agricultura familiar (Buralli et al., 2019).

Mas como reverter esse quadro? Como minimizar dados tão preocupantes? Para promover sucessivamente o desenvolvimento rural, é necessário proporcionar alternativas sustentáveis. Estratégias de produção que não dependam de insumos sintéticos e possuam o maior aproveitamento energético dentro do sistema, como por exemplo, a Agricultura Orgânica, a Agricultura Biodinâmica, a Agricultura Biológica, a Agricultura Natural, a Permacultura, a Agroecologia, entre outras (Caporal \& Azevedo, 2011). E que o modelo industrial de produção de alimentos seja repensado, para que assim, hajam opções menos prejudiciais para saúde humana e do meio ambiente, obtendo novas possibilidades na construção de um novo 
paradigma do uso sustentável do espaço rural, proporcionando melhores perspectivas de vida.

\section{Considerações Finais}

Pesquisas com esse objetivo são fundamentais para contextualizar os assuntos, pois a metodologia adotada ajuda a construir uma interface completa, da base de literatura até as novidades científicas de uma determinada problemática. Os fatores de risco que os agricultores familiares estão sujeitos podem ser tanto a curto quanto a longo prazo, dependendo do contato, frequência e quantidade. Além de doenças e reações, o perigo também pode ocorrer através da facilidade ao acesso e as crises de transtornos psicológicos.

Neste âmbito, é importante ressaltar que os trabalhadores rurais familiares possuem uma maior fragilidade, decorrentes de fatores socioeconômicos, baixa escolaridade e ausência de suporte técnico. Contudo, isso não significa considerar que estes tenham pouco conhecimento, pois, essas pessoas têm uma gama encorpada e aprofundada no que tange produção agrícola, entretanto estes saberes não abrangem noções técnicas a respeito dos agrotóxicos, sendo necessário maior suporte do poder público e empresas privadas para esses agricultores. Deve haver mais informação aos agricultores, em relação aos usos, à toxidade e manuseio. Entretanto, uma alternativa que mudaria esse panorama, seria a adoção de sistemas orgânicos de produção (utilizando manejos alternativos no controle de pragas, doenças e plantas espontâneas, adubação).

Ademais, as reflexões trazidas podem orientar a criação de políticas públicas e medidas de fiscalização que possam fornecer suporte técnico e treinamento adequado a respeito da aplicação de forma segura de agrotóxicos e esclarecer sobre os impactos danosos dos agroquímicos à saúde humana e ao meio ambiente. Nesse âmbito, também é essencial que as possíveis providências possam ser fundamentadas em abordagens integradas, que considerem o contexto socioeconômico, ambiental e de saúde, em que estes então inseridos.

Para um aperfeiçoamento dessa análise, é importante que novos estudos sejam realizados, sejam eles de ordem teórica ou prática, testando várias metodologias para que a validade contribua como alerta e incentivo à prevenção dos usos de agroquímicos, realizados de maneira indiscriminadas, sem a devida proteção, entre outras questões que podem levar à acidentes e tragédias.

\section{Agradecimentos}

Os autores agradecem à Universidade Tecnológica Federal do Paraná (UTFPR) - Campus Bato Branco, ao Programa de Pós-graduação em Desenvolvimento Regional (PPGDR) e à Coordenação de Aperfeiçoamento de Pessoal de Nível Superior (CAPES).

\section{Referências}

Abbasi-Jorjandi, M., Asadikaram, G., Abolhassani, M., Fallah, H., Abdollahdokht, D., Salimi, F., \& Pournamdari, M. (2020). Pesticide exposure and related health problems among family members of farmworkers in southeast Iran. A case-control study. Environmental Pollution, $267,115424$.

Andin, A. R. A., Ashar, T., \& Lubis, R. (2020). The Relationship between Personal Hygiene and Household Insecticide Use with Parkinsonism on Farmers at Juhar Ginting Sadanioga. Britain International of Exact Sciences (BIoEx) Journal, 2(1), 102-107.

Agência Nacional de Vigilância Sanitária. (2011). Programa de Análise de Resíduos de Agrotóxicos em Alimentos (PARA): Relatório de Atividades de 2009.

Bach, M. A., Samms-Vaughan, M., Hessabi, M., Bressler, J., Lee, M., Zhang, J., \& Rahbar, M. H. (2020). Association of polychlorinated biphenyls and organochlorine pesticides with autism spectrum disorder in Jamaican children. Research in Autism Spectrum Disorders, $76,101587$.

Barbosa, L. C. A. de (2004). Os pesticidas, o homem e o meio ambiente. UFV.

BBC. 2020. British Broadcasting Corporation. Brasil é principal mercado de agrotóxicos 'altamente perigosos'. https://www.bbc.com/portuguese/geral51597054\#: :text=O\%20Brasil\%20\%C3\%A9\%20o\%20pa\%C3\%ADs,de\%20soja\%2C\%20milho\%20e\%20algod\%C3 $\%$ A3o. 
Borges, I. M. S., Almeida, R. L. J., Fernandes, A. C. G., da Silva, S. É., de Andrade Silva, M. L., Barros, U. I. G., \& Freire, J. (2020). Family farming: analysis of sustainability through social economic and environmental indicators. Research, Society and Development, 9(4), 6.

Bohner, T. O. L., Araújo, L. E. B., \& Nishijima, T. (2013). O impacto ambiental do uso de agrotóxicos no meio ambiente e na saúde dos trabalhadores rurais. Revista eletrônica do curso de direito da UFSM, 8, 329-341.

Borsoi, A., dos Santos, P. R. R., Taffarel, L. E., \& Júnior, A. C. G. (2014). Agrotóxicos: histórico, atualidades e meio ambiente. Acta Iguazu, 3(1), 86-100.

Braibante, M. E. F., \& Zappe, J. A. (2012). A química dos agrotóxicos. Química nova na escola, 34(1), 10-15.

Buralli, R. J., Ribeiro, H., Leão, R. S., Marques, R. C., \& Guimarães, J. R. D. (2019). Data on pesticide exposure and mental health screening of family farmers in Brazil. Data in brief, 25, 103993.

Canal Rural. (2018). Quatro estados concentram quase $70 \%$ da produção de grãos do país. https://www.canalrural.com.br/noticias/quatro-estadosconcentram-quase-producao-graos-pais-67407/

Carneiro, F. F. (2015). Dossiê ABRASCO: um alerta sobre os impactos dos agrotóxicos na saúde. EPSJV/Expressão Popular.

Caporal, F. R., \& Azevedo, E. O. D. (2011). Princípios e perspectivas da agroecologia. Instituto Federal de Educação, Ciência e Tecnologia do ParanáEducação à Distância.

Cavalcante, D. L., \& Rodrigues, F. É. F. (2020). Debate sobre agrotóxicos num curso de Ensino Superior. Cadernos de Agroecologia, 15(2).

CONAB. 2018. Companhia Nacional de Abastecimento. Relatório de grãos. https://www.conab.gov.br/info-agro/safras/graos/boletim-da-safra-degraos/item/download/16780_e7a4a52ee1db76ad1a8cfda9b2343c48

Costa, L. F., \& de Paula Pires, G. L. (2016). Análise Histórica sobre a Agricultura e o Advento do Uso de Agrotóxicos no Brasil. Etic-Encontro De Iniciação Científica, 12(12).

Dallefi, N. M. S. da C., \& Siqueira, F. A. O. (2017). Os impactos ambientais produzidos pelo uso de agrotóxicos e a responsabilidade civil. Revista Jurídica Luso Brasileira, n. 2, 701-736.

Ercole, F. F., Melo, L. S. D., \& Alcoforado, C. L. G. C. (2014). Revisão integrativa versus revisão sistemática. Revista Mineira de Enfermagem, $18(1)$, 9-12.

Falcão, F., et al., (2013). Pesticide clippings. 239f. Informativo técnico - Secretaria de Vigilância e Atenção à Saúde do Trabalhador. Governo do Estado da Bahia. http://www.saude.ba.gov.br/wp-content/uploads/2017/08/Clipping_Agrotoxico_2013.pdf

FAO. 2014. Food and Agriculture Organization. The state of food insecurity in the world. http://www.fao.org/3/a-i4030e.pdf

FIOCRUZ. 2020. Fundação Oswaldo Cruz. Sistema Nacional de Informações Tóxico-Farmacológicas (SINITOX). Casos Registrados de Intoxicação Humana por Agente Tóxico e Zona de Ocorrência. Brasil, 2017. https://sinitox.icict.fiocruz.br/sites/sinitox.icict.fiocruz.br/files//Brasil9_1.pdf

Gonçalves, L. M. (2020). Avaliação de um agroecossistema em transição agroecológica (Master's thesis, Universidade Tecnológica Federal do Paraná).

INCA. 2020. Instituto Nacional do Câncer. Agrotóxicos: causas e prevenção. 2020. https://www.inca.gov.br/exposicao-no-trabalho-e-no-ambiente/agrotoxicos

IPEA. 2019. Instituto de Pesquisa Econômica Aplicada. Agrotóxicos no brasil: padrões de uso, política da regulação e prevenção da captura regulatória. http://repositorio.ipea.gov.br/bitstream/11058/9371/1/td_2506.pdf

Lopes, C. A. (2017). É possível produzir alimentos para o Brasil sem agrotóxicos? Ciência e Cultura, 69(4), 52-55.

Mehmood, Y., Arshad, M., Kaechele, H., Mahmood, N., \& Kong, R. (2021). Pesticide residues, health risks, and vegetable farmers' risk perceptions in Punjab, Pakistan. Human and Ecological Risk Assessment: An International Journal, 27(3), 846-864.

MAPA. 2018. Ministério da Agricultura, Pecuária e Abastecimento. Monitoramento agrícola 2018. https://www.conab.gov.br/info-agro/safras/graos/boletimda-safra-de-graos/item/download/1291_40bc3f850bc23ec2d6ada18c7c98be5c

MAPA. 2019. Ministério da Agricultura, Pecuária e Abastecimento. Alterações das classificações toxicológicas dos produtos formulados agrotóxicos. ATO ${ }^{\circ}$ 58, de 27 de agosto de 2019. https://www.in.gov.br/web/dou/-/ato-n-58-de-27-de-agosto-de-2019-213474289

Ministério da Agricultura, Pecuária e Abastecimento. Agricultura Familiar, 2016. Lei Federal n. 7.802, de 11 de julho de 1989. http://www.planalto.gov.br/ccivil_03/leis/17802.htm

Ministério do Trabalho e Emprego. Norma Regulamentadora 31 - Segurança e saúde no trabalho na agricultura, pecuária silvicultura, exploração florestal e aquicultura. https://www.gov.br/trabalho/pt-br/inspecao/seguranca-e-saude-no-trabalho/ctpp-nrs/norma-regulamentadora-no-31-nr-31.

Ministério da Saúde. Secretaria de Vigilância em Saúde. Departamento de Vigilância em Saúde Ambiental e Saúde do Trabalhador. Agrotóxicos na ótica do Sistema Único de Saúde / Ministério da Saúde, Secretaria de Vigilância em Saúde, Departamento de Vigilância em Saúde Ambiental e Saúde do Trabalhador. https://bvsms.saude.gov.br/bvs/publicacoes/agrotoxicos_otica_sistema_unico_saude_v2.pdf

Okuyama, J. H. H., Galvão, T. F., \& Silva, M. T. (2020). Poisoning and associated factors to death from pesticides: case-control study, Brazil, 2017. Revista Brasileira de Epidemiologia, 23, e200024.

Pavani, N. D. (2016). Pesticidas: uma revisão dos aspectos que envolvem esses compostos. Trabalho de Conclusão de Curso (licenciatura-Química) Universidade Estadual Paulista, Faculdade de Ciências. 
Research, Society and Development, v. 10, n. 9, e58510918414, 2021

(CC BY 4.0) | ISSN 2525-3409 | DOI: http://dx.doi.org/10.33448/rsd-v10i9.18414

Petarli, G. B., Cattafesta, M., Luz, T. C. D., Zandonade, E., Bezerra, O. M. D. P. A., \& Salaroli, L. B. (2019). Exposição ocupacional a agrotóxicos, riscos e práticas de segurança na agricultura familiar em município do estado do Espírito Santo, Brasil. Revista Brasileira de Saúde Ocupacional, 44.

Porto, M. F. D. S. (2018). The tragic "Poison Package": lessons for Brazilian society and Public Health. Cadernos de saude publica, 34.

Ramos, M. L. H., da Silva Lima, V., da Silva, R. E., do Nascimento Nunes, J. V., \& da Silva, G. C. (2020). Perfil epidemiológico dos casos de intoxicação por agrotóxicos de 2013 a 2017 no Brasil. Brazilian Journal of Development, 6(7), 43802-43813.

Ristow, L. P., Battisti, I. D. E., Stumm, E. M. F., \& Montagner, S. E. D. (2020). Fatores relacionados à saúde ocupacional de agricultores expostos a agrotóxicos. Saúde e Sociedade, 29, e180984.

Santana, V. S., Moura, M. C. P., \& Nogueira, F. F. (2013). Mortalidade por intoxicação ocupacional relacionada a agrotóxicos, 2000-2009, Brasil. Revista de Saúde Pública, 47, 598-606.

Schlosser, J. F., Martini, A. T., Farias, M. S. D., Bertollo, G. M., Santos, G. O. D., Barbieri, J. P., \& Negri, G. M. (2017). Regulagem, calibração, estado de conservação e uso de pulverizadores agrícolas no estado do Rio Grande do Sul. 1 ed.

Schneider, S. (2016). A presença e as potencialidades da agricultura familiar na América Latina e no Caribe. Redes: revista do desenvolvimento regional. Santa Cruz do Sul, RS. 21(3), pt. 2 (set./dez. 2016), p. 11-43.

SINDAG. 2017. Sindicato Nacional da Indústria de Produtos para Defesa Agrícola. Boletins de Estatísticas de Mercados de agrotóxicos. São Paulo, 19952007. 2017. http://www.sindag.org.br/

SINAN. 2019. Sistema de Informação de Agravos de Notificação. Normas e rotinas. DATASUS, Ministério da Saúde. 2019. http://tabnet.datasus.gov.br/cgi/tabcgi.exe?sinannet/cnv/Intoxbr.def

Silvério, A. C. P., Martins, I., Nogueira, D. A., Mello, M. A. S., Loyola, E. A. C. D., \& Graciano, M. M. D. C. (2020). Assessment of Primary Health Care for rural workers exposed to pesticides. Revista de saude publica, 54, 09.

Tittonell, P. (2020). Assessing resilience and adaptability in agroecological transitions. Agricultural Systems, 184, 102862.

Toledo, E. N. B., \& Zonin, V. J. (2020). Agricultura familiar em busca de renda monetária e a relativização da sustentabilidade. COLÓQUIO-Revista do Desenvolvimento Regional, 17(4), 140-167.

Tourinho, R., \& Portela, G. (2016). Pressão política dificulta redução do uso de agrotóxicos no Brasil. https://www.icict.fiocruz.br/content/press\%C3\%A3opol\%C3\%ADtica-dificulta-redu\%C3\%A7\%C3\%A3o-do-uso-de-agrot\%C3\%B3xicos-no-brasil

Upadhayay, J., Rana, M., Juyal, V., Bisht, S. S., \& Joshi, R. (2020). Impact of pesticide exposure and associated health effects. Pesticides in crop production: physiological and biochemical action, 69-88.

Wang, J., Xue, J., Jiang, Y., Zhu, T., \& Chen, S. (2020). Mediating effects of depressive symptoms on social support and quality of life among rural older Chinese. Health and Quality of Life Outcomes, 18(1), 1-8.

Wanderley, M. D. N. B. (2009). O agricultor familiar no Brasil: um ator social da construção do futuro. Revista Agriculturas: experiências em Agroecologia. RJ: Rio de Janeiro.

WHO. 2020. World Health Organization. Bulletin of the World Health Organization. https://www.who.int/bulletin/volumes/86/3/07-041814/en/

WHO/FAO. 2014. World Health Organization Food and Agriculture Organization of the United Nations. The International Code of Conduct on Pesticide Management. 2014. http://www.fao.org/3/i3604e/I3604E.pdf 